\title{
Cultural capital and social health: A cross sectional study
}

\author{
Mohammad Hassan Piran-Qeidary ${ }^{1}$, Seyfollah Farzaneh ${ }^{1 *}$, Abolghasem Heydarabadi ${ }^{1}$, Majedeh gholipur ${ }^{1}$ \\ 1. Humanistics school, Babol Islamic Azad University, Babol, Iran
}

Received: 22 November 2020

Accepted for publication: 26 December 2020

[EPub a head of print-11 January 2021]

Payesh: 2021; 20 (1):7-14

\begin{abstract}
Objective (s): Cultural capital is one capitals that plays an important role in lifestyle and social health of individuals and can affect quality of life. Therefore, the purpose of this study was to assess the correlation between cultural capital and social health in Tehran, Iran.

Methods: A cross sectional study was conducted on a sample of citizens in Tehran, Iran, in 2019. Multi-stage cluster sampling method was used to collect data using an standard questionnaire. Descriptive analyses were applied for exploring the data. A P value less than 0.05 was considered as statistical significance.

Results: In all 384 citizens were entered into the study. The results showed that the mean social health among the citizens of Tehran was equal to 2.87, which was slightly lower than average. Among the components of social health, social adaptation with 3.10 had the highest and the social participation with 2.66 had the lowest average. Only $40 \%$ of participants reported the level of cultural capital as much and very much. There was a positive and direct relationship between cultural capital and social health (Pearson correlation coefficient was 0.417 ).

Conclusion: The findings indicated that the average score of social health among the citizens of Tehran was equal to the average score and was positively correlated with cultural capital.
\end{abstract}

Key Words: social health, cultural capital, Tehran

\footnotetext{
* Corresponding author: Humanistics school, Babol Islamic Azad University, Babol, Iran

E-mail: imperpiran@gmail.com
} 


\section{نقش سر مايه فر هنكى در سلامت اجتماعى: مطالعه موردى شهروندان مناطق Y F كانه تهران}

محمد حسن يِيران قيدارى'، سيف اله فرزانه '، ابوالقاسم حيدر آبادى'، ماجده قلى يور'

1. دانشكده علوم انسانى، دانشكاه آزاد اسلامى واحد بابل، بابل، ايران

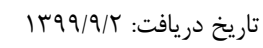

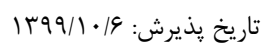

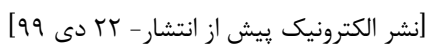

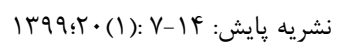

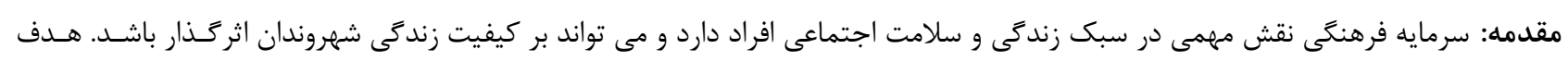

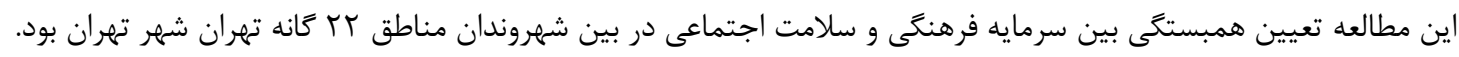

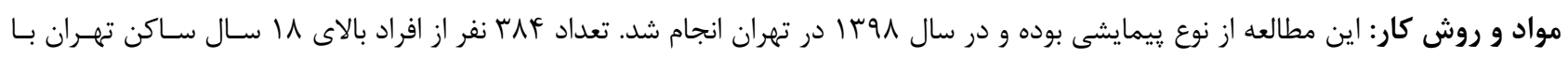

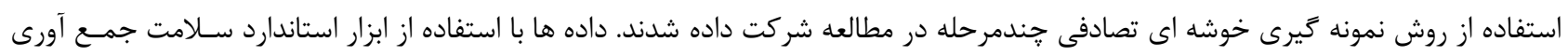

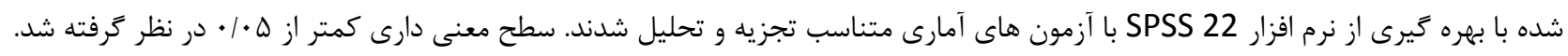

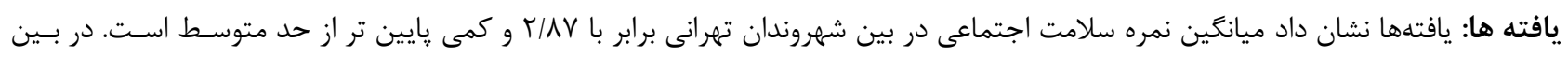

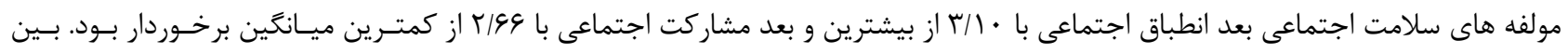

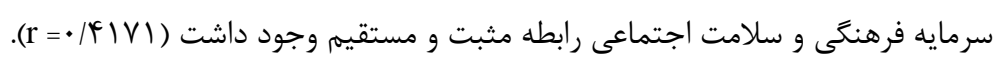

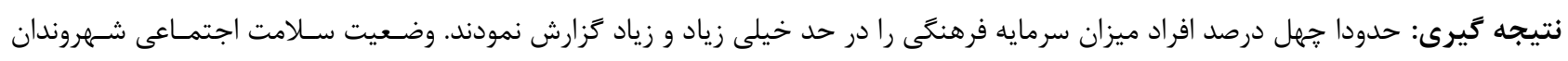

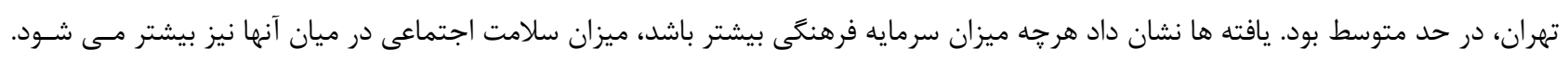

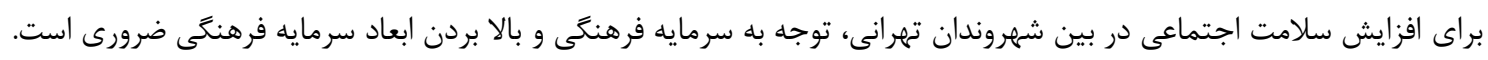


شمارى است كه شناختن آنهـا جهـت ارتقـاى سـلامت شهـروندان

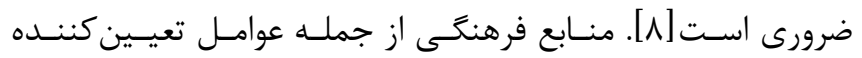

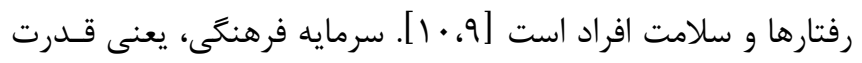

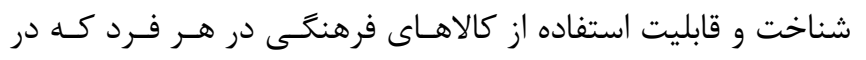

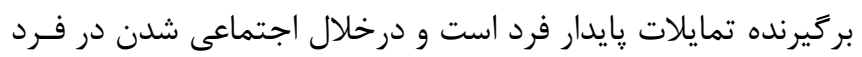

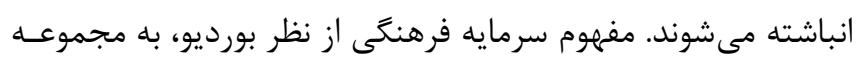

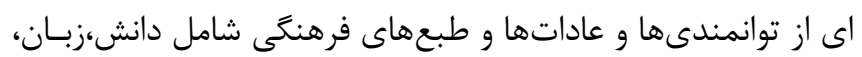

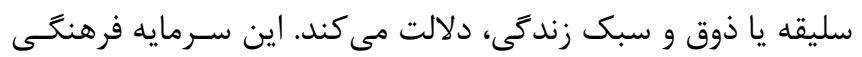

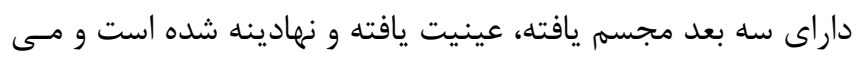
تواند بر سلامت تاثير كذار باشد [1 [1]

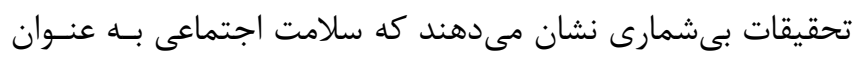

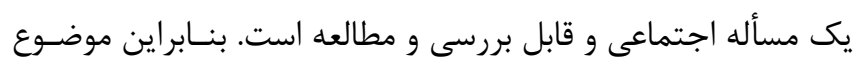

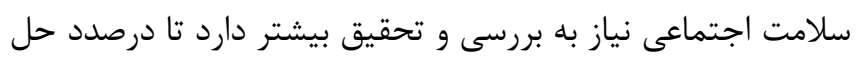

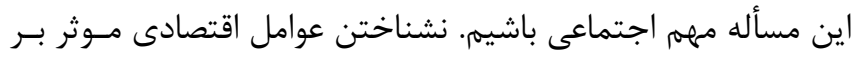

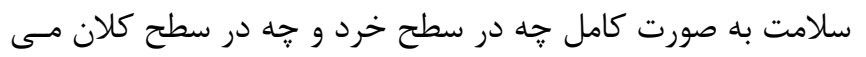

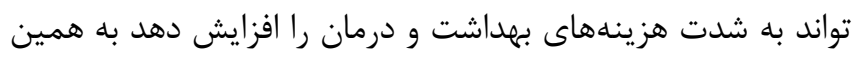

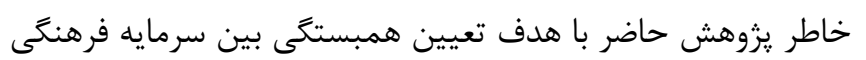

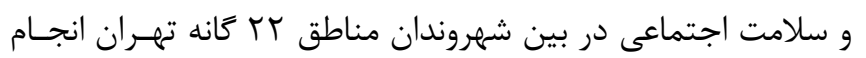

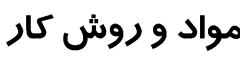

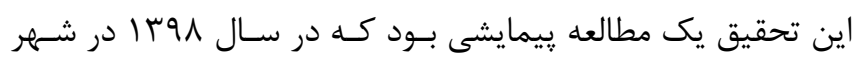

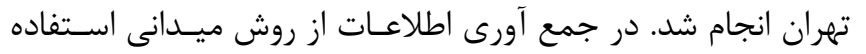

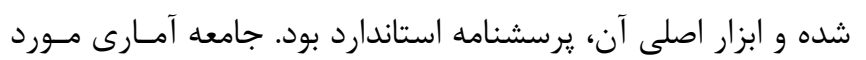

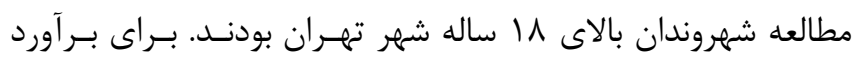

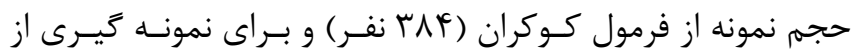

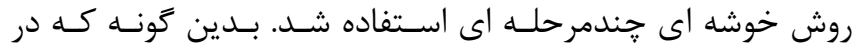

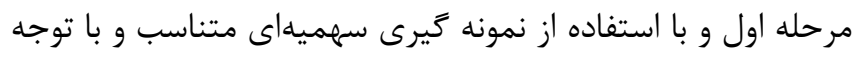

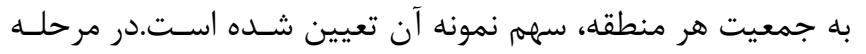

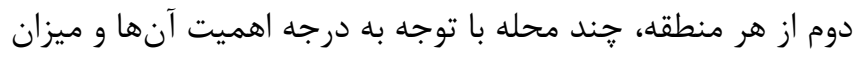

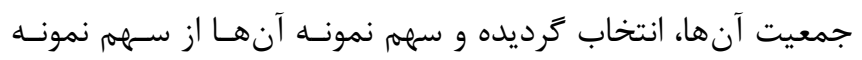

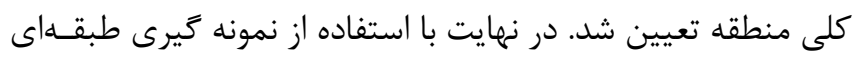

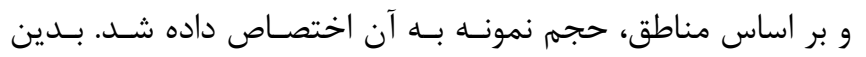

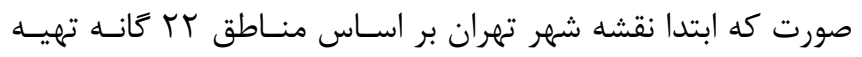

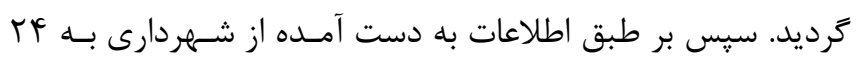
خوشه تقسيم بندى كرديد. در ادامه هر كدام از خوشه ها به شهرى

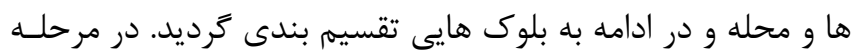

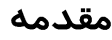

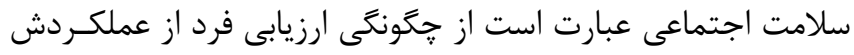

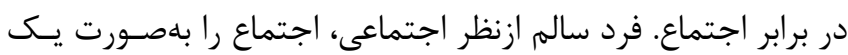

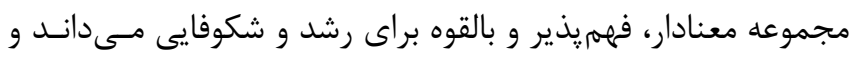

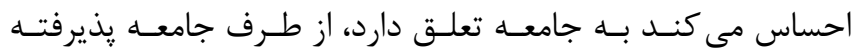

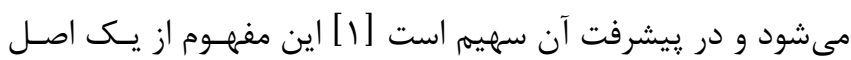

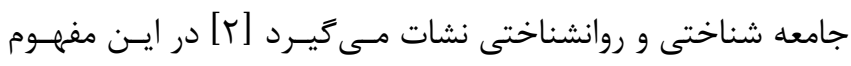

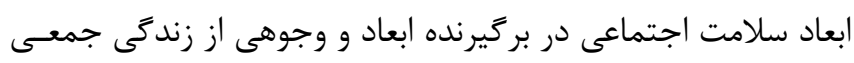

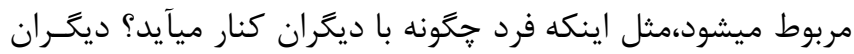

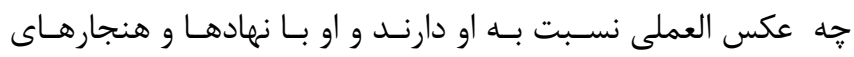

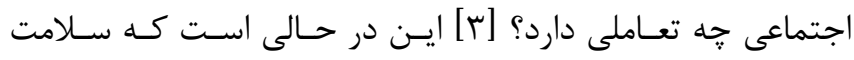

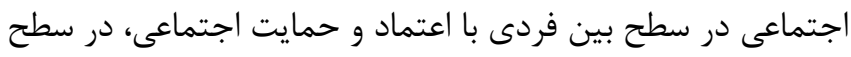

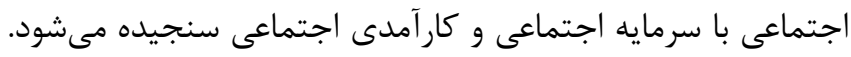

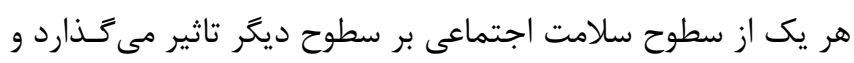

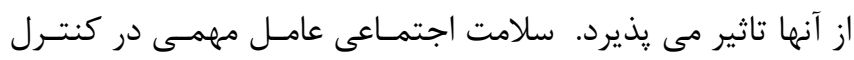

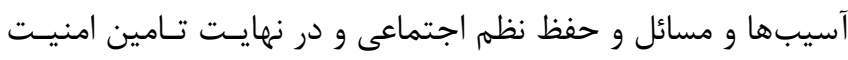

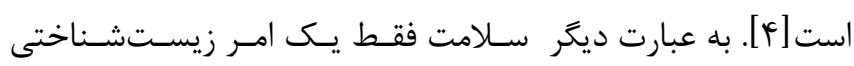

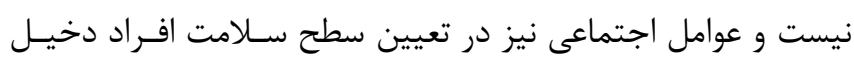

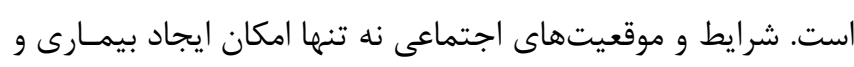

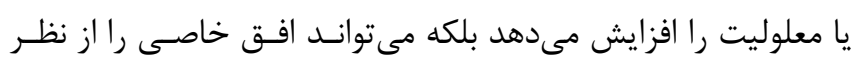

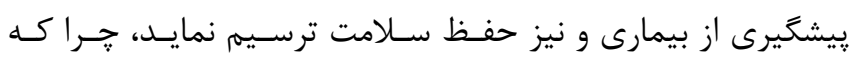

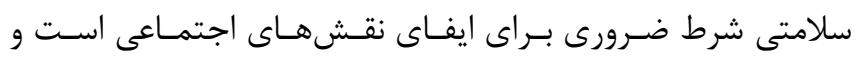

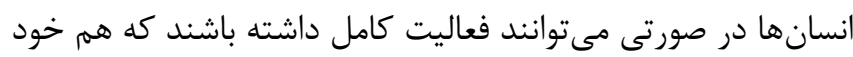

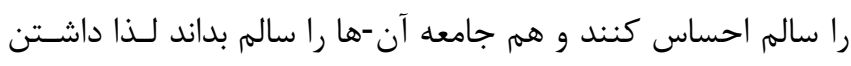

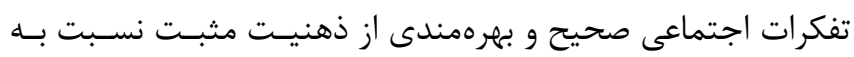

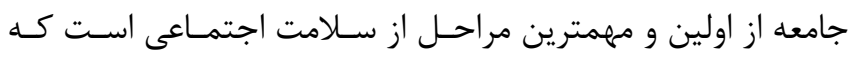

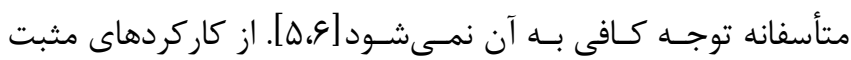

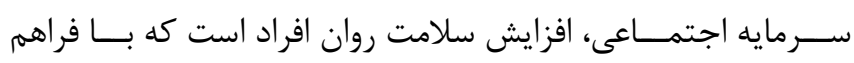

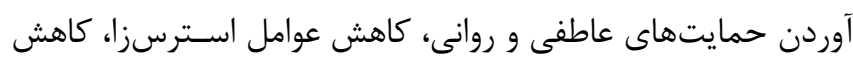
حوادث زندكَى را به همراه دارد.

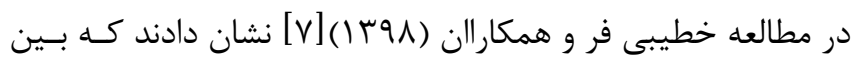

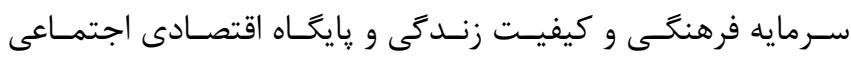

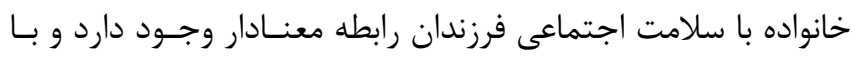

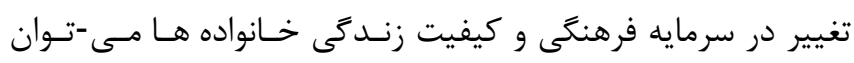

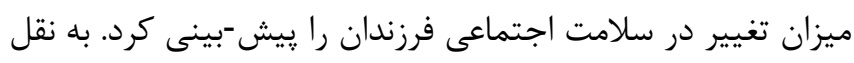

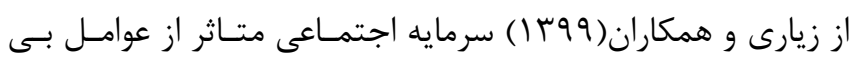


Keys بر اساس بعد اجتماعى و سـطح تحليـل فـردى، هــنج بعـد و

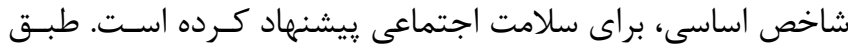

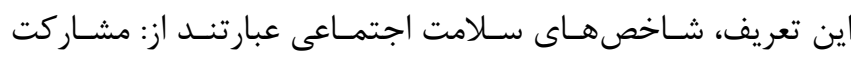

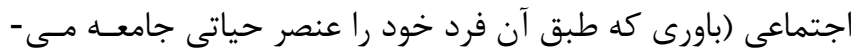

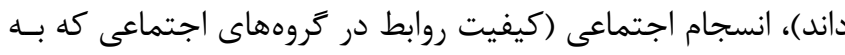

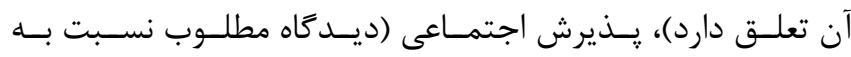

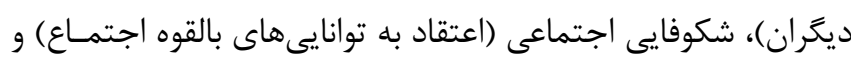

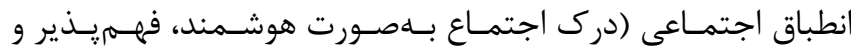
بيشبينى شونده).

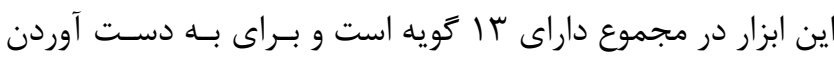

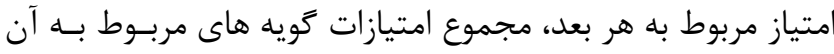

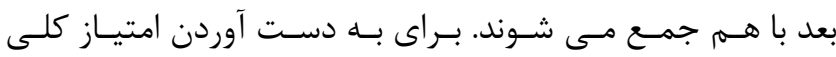

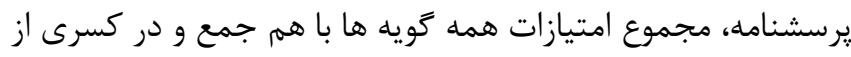

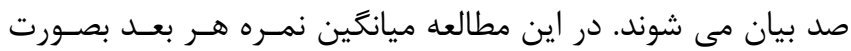

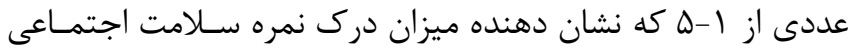

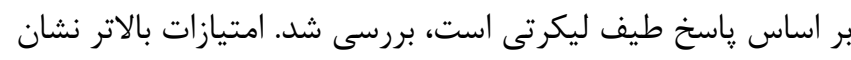

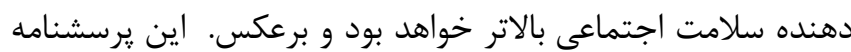

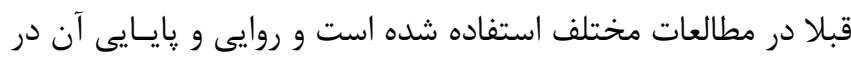

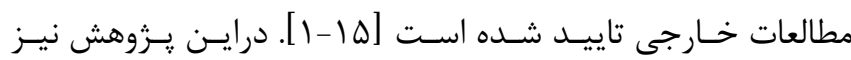
رضايت بخش كزارش شد (جدول (1).

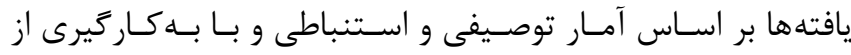

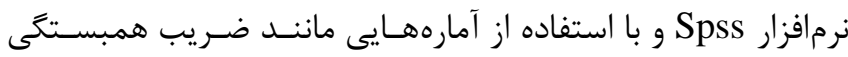

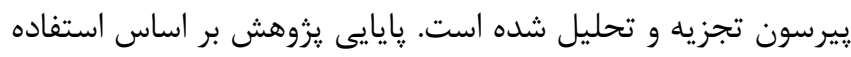

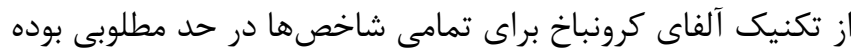

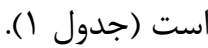
از كليه شركت كنندكان رضايت نامه آكاهانه اخذ شد.

\section{يافتهها}

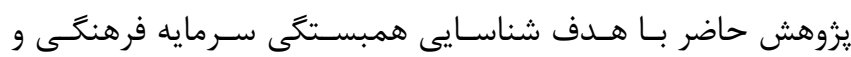

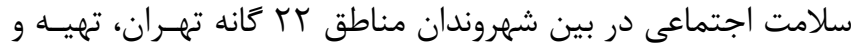

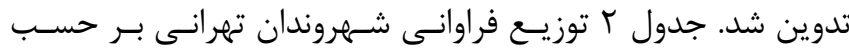

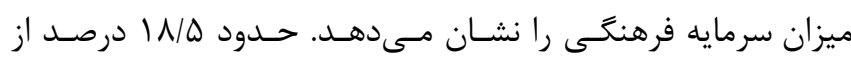

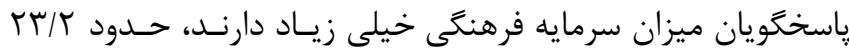

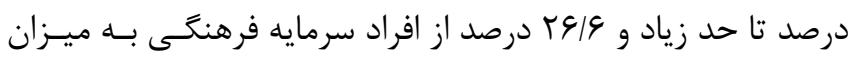

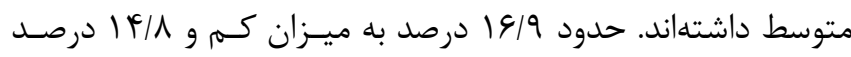
نيز سرمايه فرهنكى در حد خيلى كم را كزارش نمودند.
بعد با استفاده از نمونه گيرى تصادفى از هـر خوشـه، از بـين محلـهـ هاى مشخص شده يك محله كه معرف كل خوشه باشد انتخاب شد.

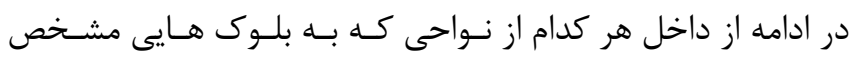

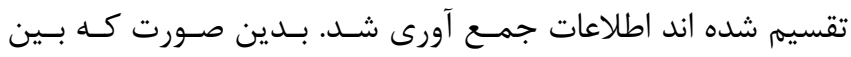
فهرست بلوك هاى هر قسمت تعيين شده، تعدادى بلوك (به تعـداد

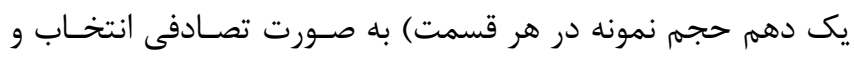

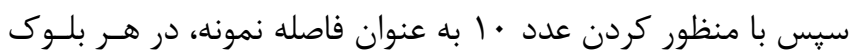

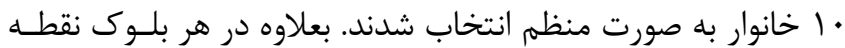

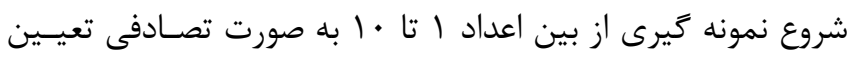

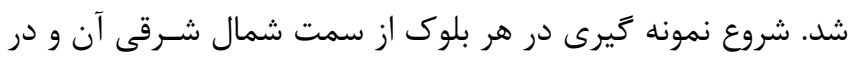
جهت حركت عقربه ساعت صورت كرفته و بلوك هايى كـه كمتـــ از

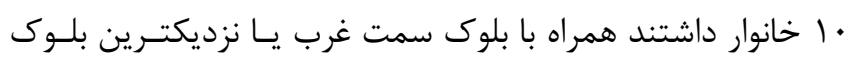
مسكونى به آنها در نظر كرفته شدند.

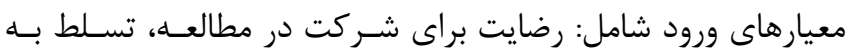
زبان فارسى و سواد خواند و نوشتن بودند.

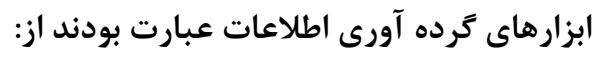

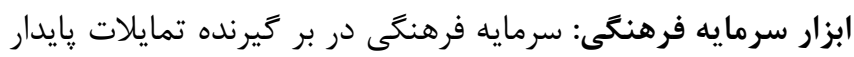
فرد است يعنى شامل كرايشها و عاداتى است كه در سرمايه فرهنتى

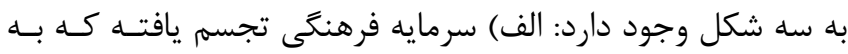

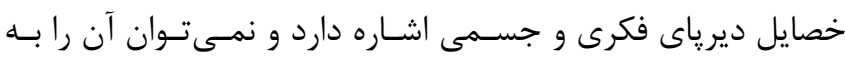

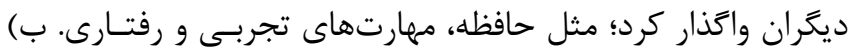

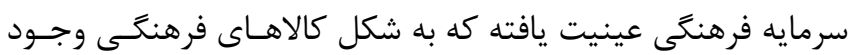

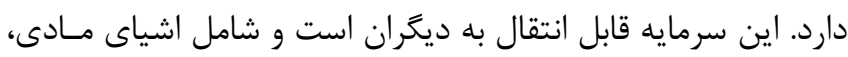

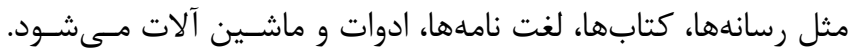
ج) سرمايه فرهنكى نهادينه شده كه به كمك ضوابط اجتماعى و بـهـ

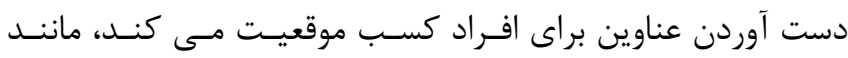

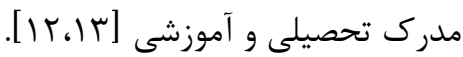

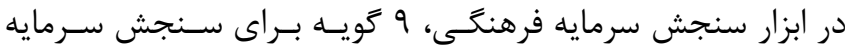

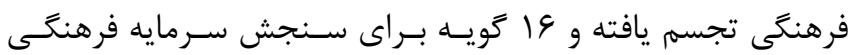

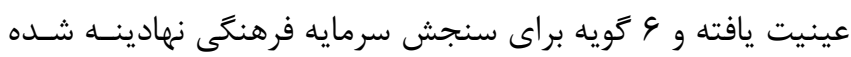

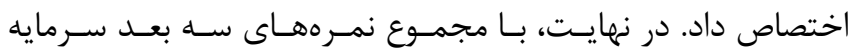
فرهنكى (تجسم يافته، عينيت يافته و نهادينه شــده)، نمـره سـرمايه فرهنكى كل نيز به دست آمده است ابزارسلامت اجتماعى: سلامت اجتماعى، ارزيابى و شناخت فـرد از

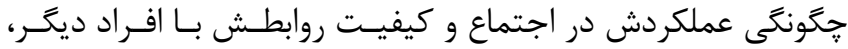

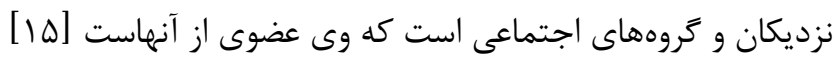


جدول أ، رابطه بين سرمايه فرهنگى و سلامت اجتمــاعى آنـان (بــه

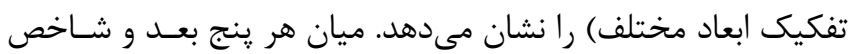

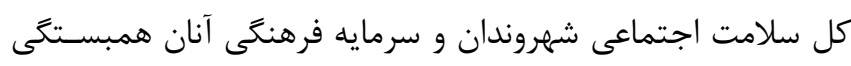
مثبت و معنادار (در سطح ه • • درصد)، وجود دارد.

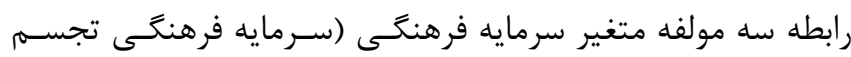

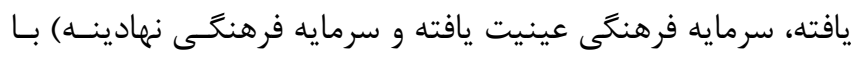

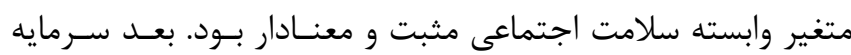

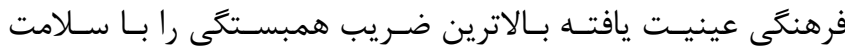

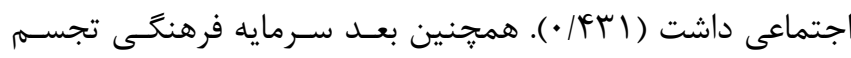

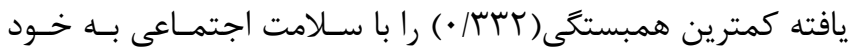

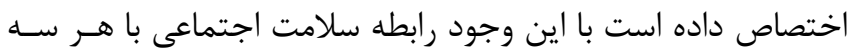
بعد سرمايه فرهنگى مثبت و معنادار و در حد پايين بود (جدول ه).
جدول r توزيع شهروندان بر حسب ابعاد سلامت اجتماعى را نشـان مى دهد. بيشترين مقدار مربوط بـهـ متوسـط يـا تاحـدودى سـلامت

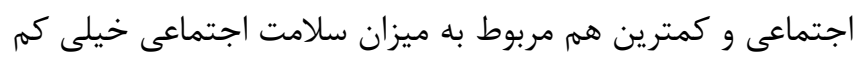

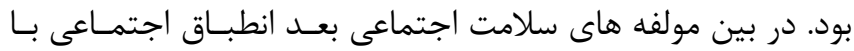

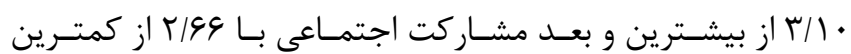
ميانگين برخوردار بود (جدول ب).

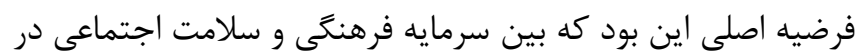
بين شهروندان مناطق r T Fانه تهران همبستگى معنادار وجود دارد.

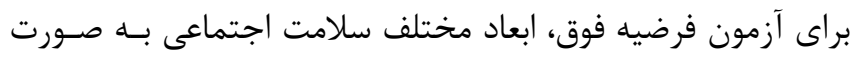

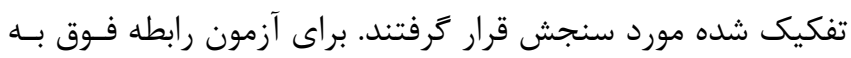
علت نرمال بودن و برخوردارى از سطح سنجش فاصـلهاى متغيرهـا، از آزمون ضريب همبستخى پيرسون استفاده شد.

جدول ا: ضرايب آلفاى كرونباخ و پايايى متغيرها

\begin{tabular}{|c|c|c|c|}
\hline \multicolumn{2}{|c|}{ ضريب آلفاى كرونباخ } & \multirow[b]{2}{*}{ ابعاد اد } & \multirow[t]{2}{*}{ متغيرهاى يُزوهش } \\
\hline 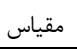 & خرده مقياس & & \\
\hline \multirow{3}{*}{$\cdot|\Lambda|$} & $\cdot / v 9$ & سرمايه فرهنگى تجسم يافته & \multirow{3}{*}{ سرمايه فرهنَى } \\
\hline & $\cdot \operatorname{lAt}$ & سرمايه فرهنكَى عينيت يافته & \\
\hline & $\cdot \mid M F$ & سرمايه فرهنگَى نهادينه شده & \\
\hline \multirow{5}{*}{$\cdot / V \wedge$} & $\cdot / \Lambda V$ & يكيار جكى اجتماعى & \multirow{5}{*}{ سلامت اجتماعى } \\
\hline & $\cdot / V T$ & يذيرش اجتماعى & \\
\hline & $\cdot \mid \Lambda \Lambda$ & مشاركت اجتماعى & \\
\hline & $\cdot / 9 V$ & شكوفايى اجتماعى & \\
\hline & .199 & انطباق اجتماعى & \\
\hline
\end{tabular}

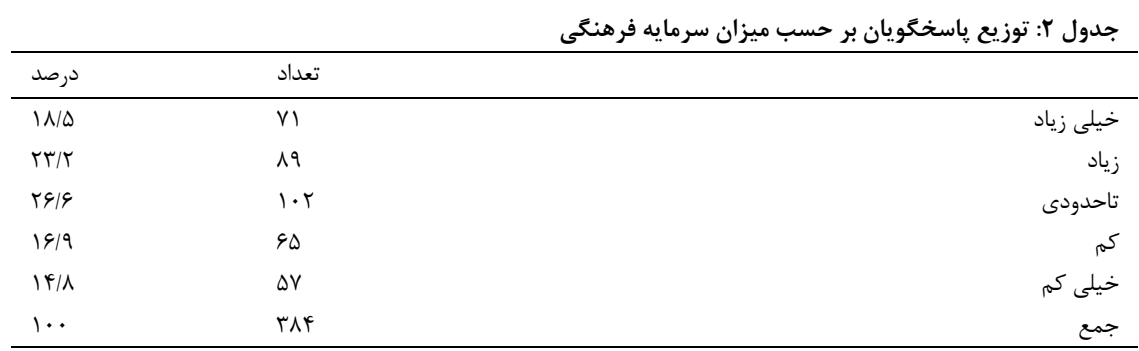

جدول r: توزيع ياسخكَويان بر حسب ميزان سلامت اجتماعى

\begin{tabular}{|c|c|c|c|c|c|c|c|c|c|c|c|c|c|}
\hline \multirow{3}{*}{ 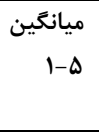 } & \multirow{2}{*}{\multicolumn{2}{|c|}{ 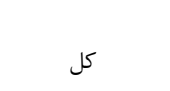 }} & \multirow{2}{*}{\multicolumn{2}{|c|}{ خيلى كم }} & \multirow{2}{*}{\multicolumn{2}{|c|}{ كم }} & \multirow{2}{*}{\multicolumn{2}{|c|}{ متوسط }} & \multicolumn{5}{|c|}{ توزيع پاسخ ها } \\
\hline & & & & & & & & & & & & خيل ل & \\
\hline & درصد & فراوانى & درصد & فراوانى & درصد & فراوانى & درصد & فراوانى & درصد & فراوانى & درصد & فراوانى & \\
\hline$r / 91$ & $1 \cdots$ & rNF & $\mid F / r$ & $\Delta \Delta$ & $r \cdot 19$ & v9 & $r 4 / q$ & ITF & $r \mu / T$ & 19 & $\mathrm{v}$ & tr & انسجام اجتماعى \\
\hline$r / 9 V$ & $1 \cdots$ & rNF & $r \cdot / 1$ & VV & $r \cdot / \Lambda$ & $\wedge \cdot$ & rT/G & IrD & $\mid f / 9$ & $\Delta \varphi$ & ir & is & ذِيرش اجتماعى \\
\hline$r / 94$ & $1 \cdots$ & MAF & $T r / V$ & $\wedge \vee$ & $19 / 0$ & va & $19 / 0$ & $1 \cdot v$ & $1 N / \Lambda$ & VT & $11 / r$ & er & مشاركت اجتماعى \\
\hline$r / 1$. & $1 \cdots$ & rNF & $1 \cdot / V$ & il & $10 / 9$ & c. & $r M / r$ & $\pi$ & TF & 94 & $1 N / \Delta$ & vi & انطباق اجتماعى \\
\hline$r / \cdot r$ & $1 \cdots$ & rNF & $11 / r$ & pr & $19 / 0$ & VQ & r & 149 & $T F / D$ & $9 f$ & $\Lambda / 9$ & 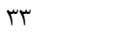 & شكوفايى اجتماعى \\
\hline$r / A V$ & $1 \cdots$ & MAF & $r \cdot / r$ & $\vee \wedge$ & $r r / r$ & 19 & $r \Delta / \Delta$ & 91 & $r \cdot 19$ & V9 & $1 \cdot 14$ & f. & سلامت اجتماعى \\
\hline
\end{tabular}


سال بيستم، شماره اول، بهمن ـاسفند 99

نشريه يروهشكده علوم بهداشتى جهادانشكاهى

جدول f: آزمون ضريب همبستكى بيرسون بين سرمايه فرهنكَى و سلامت اجتماعى

\begin{tabular}{|c|c|c|c|}
\hline سطح معنى دارى & ضريب بيرسون & متغير وابسته & متغير مستقل \\
\hline$\cdot / \cdot .1$ &.$/ 411$ & شكوفايى اجتماعى & سرمايه فرهنكى \\
\hline $.1 . .1$ & grra & انطباق اجتماعى & \\
\hline$\cdot 1 . .1$ & . & يذيرش اجتماعى & \\
\hline $.1 . .1$ & . MtT & مشاركت اجتماعى & \\
\hline $.1 . .1$ & $\cdot / 4 \cdot r$ & انسجام اجتماعى & \\
\hline $.1 . .1$ &.$/ F I V$ & سلامت اجتماعى & \\
\hline
\end{tabular}

\begin{tabular}{|c|c|c|c|}
\hline \multicolumn{4}{|c|}{ جدول ه: ضريب همبستكَى ييرسون بين ابعاد سر مايه فرهنكَى و سلامت اجتماعى } \\
\hline سطح معنى دارى & ضريب همبستكى يِيرسون & متغير وابسته & متغير مستقل \\
\hline$\cdot 1 \cdot .1$ & 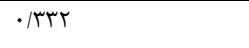 & 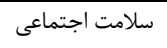 & سرمايه فرهنكى تجسم يافته \\
\hline$\cdot 1 \cdot .1$ & $\cdot \mid q 41$ & & سرمايه فرهنكَى عينيت يافته \\
\hline$\cdot / \cdot 1$ & $\cdot / 4 \cdot \Delta$ & & سرمايه فرهنكَى نهادينه شده \\
\hline
\end{tabular}

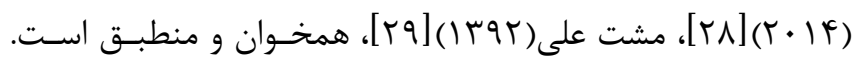

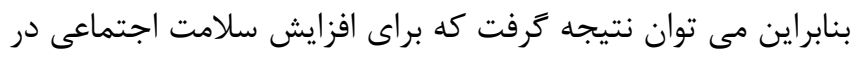

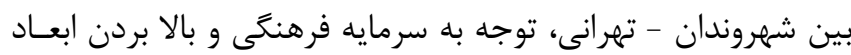

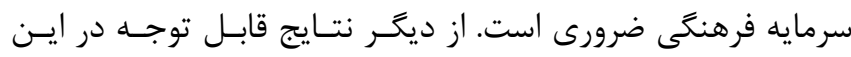

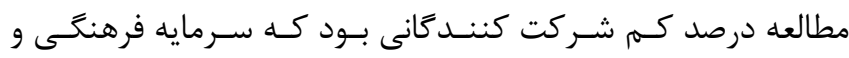

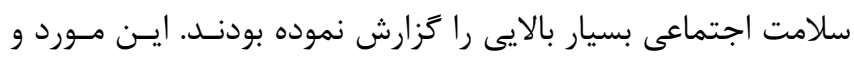

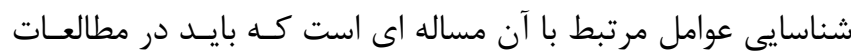

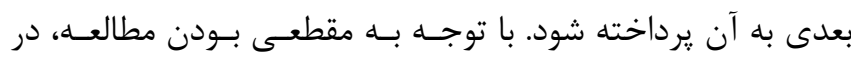

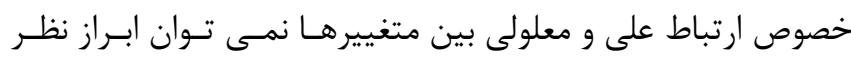

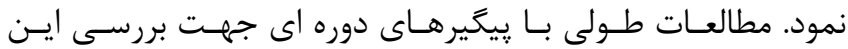
ارتباطات توصيه مى شود.

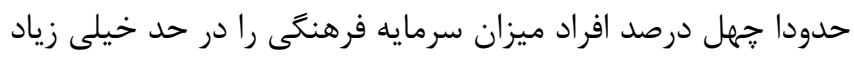
و زياد كزارش نمودند. وضعيت سلامت اجتماعى شهروندان تهـران،

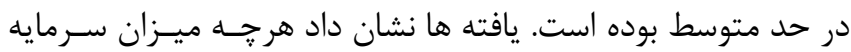

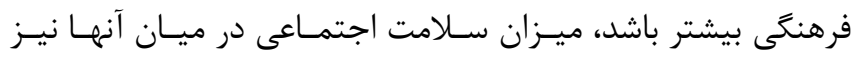

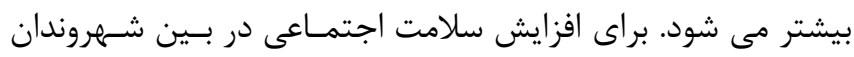

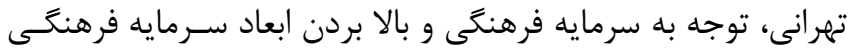

$$
\text { ضرورى است. }
$$

\section{سهم نويسند}

محمدحسن ييران: دانشجو، طراحى و اجراى مطالعه ، نوشتن مقاله سيف اله فرزانه: استاد راهنما، نوشتن مقاله ابوالقاسم حيدرابادى: استاد مشاور، نوشتن مقاله ماجده قلى يور: استاد مشاور، نوشتن مقاله

تشكر و قدردانى از كليه شركت كنندكان يزوهش تشكر و قدردانى مى شود.

\section{بحث و نتيجه گيرى}

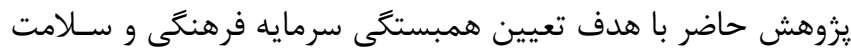

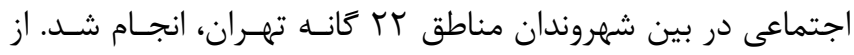

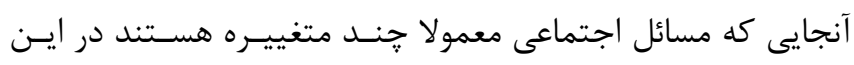

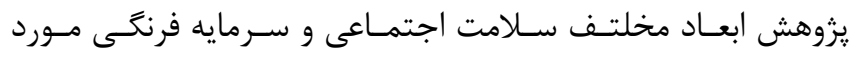
بررسى قرار كرفتند ـ نتايج نشان داد كه بين ابعاد سلامت اجتماعـى

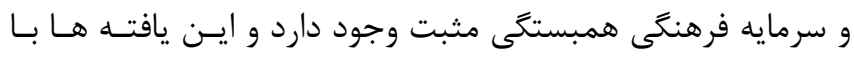

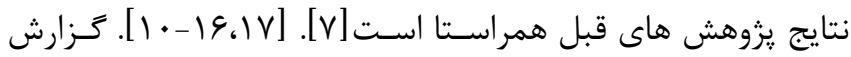

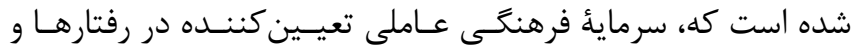

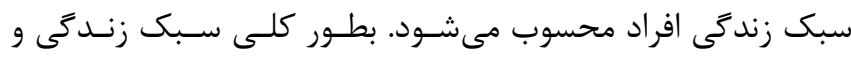

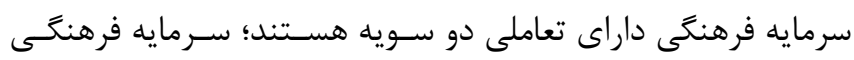

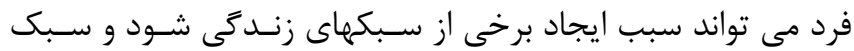

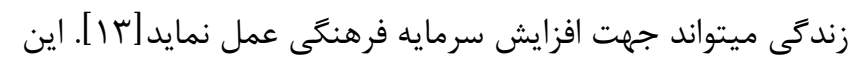

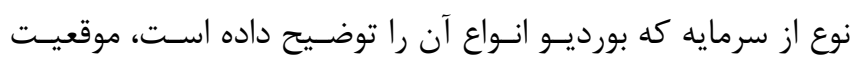

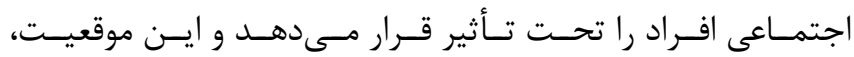

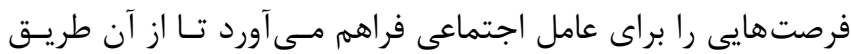

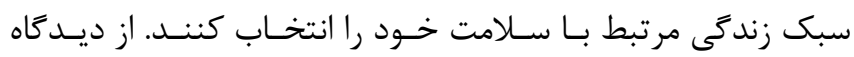

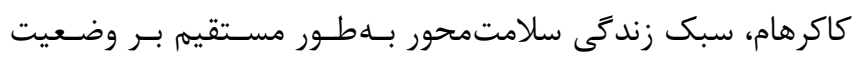

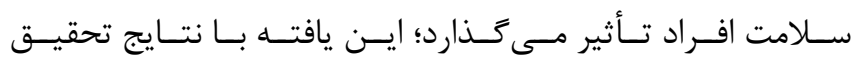

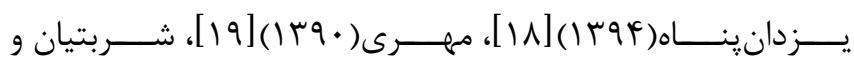

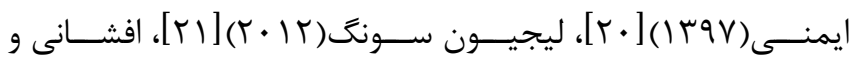

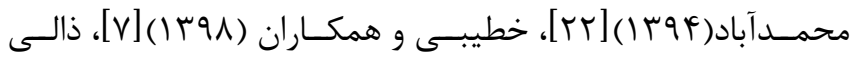

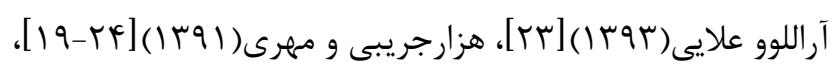

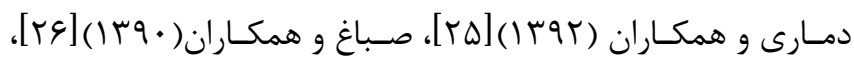

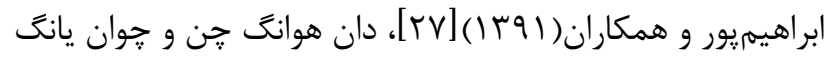




\section{منابع}

1. Keyes C. L. M. "Mental Health and / or Mental Illness? Investigation Axioms of the Complet state model of Health". Jornal of Consulting and Clinicial Psychology 2004;73: 539-548 [Persian]

2. Mosavi M ,Shiani M,fateminia M,Omidnia S, Conceptual development of social capital with a social health approach. Social Welfare 2015;57:109-150 [Persian]

3. Tavakol M. Social Health: Dimensions, Components, and Indicatorsin Iranian and World Studies. Journal of Bioethics 2015; 14:115-135 [Persian]

4. Samaram E, community-oriented policing and social health. Entezam-e-Ejtemaei 2009; 1:9-29 [Persian]

5. Talebi A, Mohammadi A. Investigating the social factors affecting the social health of Dehaghan Azad University students. International Journal of Nations Research 2020;51:130-148 [Persian]

6. Sabbagh S, Moinian M, Sabbagh S. The assessment of the social factors associated with social health of families in Tabriz. Sociological Studies 2011;10:27-44 [Persian]

7. Khatibi A, RajabiFar. The Relationship between Cultural Capital and Family Quality of Life with Social Health of Students in Marivan City. Scientific Quarterly of the Education Society 2020; 12:118-122 [Persian]

8. ziari K, Pourahmad A, Hatami Nejad H, Mahdi A. Analysis of the urban social health from the perspective of social capital in the metropolises of the Iranian religious Case study: Metropolis Qom. Journal of Spatial Planning 2020;35:51-66. [Persian]

9. Reza Salehi Amiri. Cultural capital. $2^{\text {th }}$ Edition, Strategic Research Center:Tehran 2012 [Persian]

10. Ghaderi M, Maleki A, Ahmadnia Sh. from bourdieus cultural capital to abels health-related cultural capital: applying new indicators for explaining healthy lifestyle. Social Welfare 2016;16:9-58 [Persian]

11. Naser fakohi. History of Anthropological Thought and Theories, 12 ${ }^{\text {th }}$ Edition, nashr ny: Tehran 2002 [Persian]

12. Akbarzadeh F, Dehghani H, Khoshfar G, Janalizadeh $H$. The Impact of Three Types of Economic, Cultural and Social Capitals on Youth's Happiness. Applied Sociology 2013;2:88-67 [Persian] 13.Gamal M,Vadad hir A, Mohamadi F.Cultural Capital Relations and Lifestyle: A Survey of MiddleClass Citizens in Sanandaj, Kurdistan. Iranian Journal of Sociology 2012;4:13-23 [Persian]

14. Bourdieu, Pierre. Raisons pratiques: sur la theorie de l'action, $3^{\text {th }}$ Edition. Role and Likeness: Tehran 2001
15. Keyes CLM, Shapiro AD. Social Well-Being in the United States: A Descriptive Epidemiology. How healthy are we?: A national study of well-being at midlife. The John D. and Catherine T. MacArthur foundation series on mental health and development. Studies on Successful Midlife Development. Chicago, IL, US: The University of Chicago Press 2004

16. Hedarkhani $h$,Haghiaghaee L , Rahmani G. the relationship between body shape and management of cultural capital among women. Women and Cultuorer 2014;18:55-69

17. Esfahani M, Mahmoudian H, A Study of the Impact of Cultural Capital on General Health by the Mediating role of Healthy Lifestyle (Case study: High School Students of Shiraz). Journal of Applied Sociology2018;2:1-16 [Persian]

18. Panah Yazdan L, Nikvarz T. Relationship between social factors and social health of students of Shahid Bahonar University of Kerman. Journal of Applied Sociology $2015 ; 59: 99-116$ [Persian]

19. Mehre Asadolah (2011), "Assessing the effect of social capital on mental and social health of teachers in Hamadan, Master Thesis in Social Welfare Planning, Supervisor: Dr. Jafar Hezarjaribi. Faculty of Social Sciences and Communication, Allameh Tabatabaei University, Tehran [Persian]

20. Sharbatiyan M,Emani N, a sociological analysis of social health of youth and factors affecting it (case study: 18- to 30-year-old citizens of qaen city). Journal of Applied Sociology 2018;69:166-188 [Persian]

21. Song, Lijun. social capital and psychological distress. Journal of Health and Social Behavior 2011;59:478-492

22. Afshani SA, ShiriMohammadabad H. The study of relationship between life satisfaction and social health of Yazd city women. Journal of Toloo-e-Behdasht yazd 2016;2:34-44 [Persian]

23. Zali Arallou M, alaei M. The study of the rate of social health among primary school teachers in Ardabil and social factors associated to it. Journal of Sociology Studies 2014;22:123-140 [Persian]

24. Hezarjaribi, G. Asadullah M.Analysis of the relationship between social capital and mental and social health. Journal of Social Sciences Allameh Tabatabai University 2012;59:41-88 [Persian]

25. Damari B , Nasehi A, VosoughMoghaddam A. What should we do for improving Iranian social health? Situational analysis, national strategies and role of ministry of health and medical education. 
Journal of School of Public Health and Institute of Public Health Research 2013;1:45-85 [Persian]

26. Sabbagh $S$, Moeinian $N$, Sabbagh $S$. the assessment of the social factors associated with social health of families in Tabriz. Journal of Sociology Studies 2011;10:27-44 [Persian]

27. Ebrahimpoor D, Pourjebelli R, Ramazanimamajani R. The Relation of Socio-Economic Factors With Social Health Of Guidance And High School Teachers Of Azarshahr City. Journal of Sociology Studies 2012;14:47-67 [Persian]
28. Danhong Chen, Tse-Chuan Yang. "The pathways from perceived discrimination to self-rated health: An investigation of the roles of distrust, Social Capital and Health Behaviors". Social Science \& Medicine 2014;104:64-73

29. Mashtali, Roghayeh (2013), Investigating the relationship between social capital and social health of students of Payame Noor University in Khoy, Master Thesis in Sociology, Payame Noor University of Tehran. Faculty of Social Sciences [Persian] 